\title{
Rewriting Logic Semantics and Verification of Model Transformations
}

\author{
Artur Boronat ${ }^{1}$, Reiko Heckel ${ }^{1}$, and José Meseguer ${ }^{2}$ \\ ${ }^{1}$ Department of Computer Science, University of Leicester \\ \{aboronat, reiko\}@le.ac.uk \\ ${ }^{2}$ Department of Computer Science, University of Illinois at Urbana-Champaign \\ meseguer@uiuc.edu
}

\begin{abstract}
Model transformations are used in model-driven development for mechanizing the interoperability and integration among modeling languages. Due to the graph-theoretic nature of models, the theory of graph transformation systems and its technological support provide a convenient environment for formalizing and verifying model transformations, which can then be used for defining the semantics of modelbased domain-specific languages. In this paper, we present an approach for formalizing and verifying QVT-like transformations that reuses the main concepts of graph transformation systems. Specifically, we formalize model transformations as theories in rewriting logic, so that Maude's reachability analysis and model checking features can be used for verifying them. This approach also provides a new perspective on graph transformation systems, where their formal semantics is given in rewriting logic. All the ideas presented are implemented in MOMENT2. In this way, we can define formal model transformations in the Eclipse Modeling Framework (EMF) and we can verify them in Maude. We use a model of a distributed mutual exclusion algorithm to illustrate the approach.
\end{abstract}

Keywords: Model and graph transformations, MOF, QVT, rewriting logic, reachability analysis, LTL model checking, Maude.

\section{Introduction}

Model transformations are used in model-driven development for mechanizing the interoperability and integration among modeling languages. Due to the graph-theoretic nature of models, the theory of graph transformation systems and its technological support provide a convenient environment for formalizing and verifying model transformations [1], which can then be used for defining the semantics of model-based domain-specific languages [2. In this work, we provide an executable formalization of QVT-like model transformations for MOF metamodels in rewriting logic, where such transformations can be executed and analyzed by model checking of invariants and of temporal logic properties.

This work should be placed within the context of current formal and informal approaches to model transformations, and can be viewed as a contribution

M. Chechik and M. Wirsing (Eds.): FASE 2009, LNCS 5503, pp. 18 -33. 2009.

(C) Springer-Verlag Berlin Heidelberg 2009 
to bringing such formal and informal approaches considerably closer within the MOF standard [3. Among the various informal approaches for model transformations (see, e.g., 44), the QVT standard [5] is probably one of the most widely accepted and has the advantage of extending MOF. However, tool support for QVT is currently only partial and the support for analyzing model transformations is still very limited. Among the formal approaches, the most widely used are based on graph transformations [6], where just a subset of the MOF modeling constructs can be directly dealt with. To the best of our knowledge, the Tiger EMF Transformation tool (EMT) 7 is the single representative tool providing termination and confluence analysis for model transformations. There is no tool support yet for model checking model transformations.

A model transformation $\beta$ can be either endogenous to a given metamodel, so that if $M: \mathscr{M}$, then $\beta(M): \mathscr{M}$, or exogenous, i.e., it can transform a model $M$ : $\mathscr{M}$ into a corresponding model $\beta(M): \mathscr{M}^{\prime}$ in a target metamodel $\mathscr{M}^{\prime}$ that need not be equal to $\mathscr{M}[8$. Furthermore, endogenous or exogenous transformations can be either functional, so that a single transformed model $\beta(M)$ is obtained, or relational, so that $\beta(M)$ is not unique, but belongs to a set of models in $\mathscr{M}^{\prime}$ obtained from a single model $M$ in $\mathscr{M}$. In this paper we focus on endogenous, relational transformations although it extends to the exogenous case easily.

At the semantic level we show how the algebraic semantics for MOF in MOMENT2 910] is extended to a rewriting logic semantics [11] of model transformations. This rewriting logic semantics is the algebraic counterpart of graph transformation systems, in the sense that: (i) models viewed as graphs correspond to the same models viewed as terms of an algebraic data type representing a given metamodel; and (ii) graph transformation rules correspond to rules in rewrite theories over these algebraic representations. Models are represented by terms that satisfy structural semantic properties as explained in Section 3.1, which are preserved by model transformations. Conceptually, this work provides interesting new connections between graph grammars and rewriting logic, not just for standard graphs, but for graphs corresponding to models: on the one hand, the fact that node attributes in graphs often contain data belonging to sophisticated algebraic data types is seamlessly supported by the rewriting logic approach while most of other approaches leave attribute values out of the formal framework; and on the other hand, rewriting logic's crucial distinction between equations and rules can be transferred to the world of graph grammars. This can be used as a powerful state reduction technique for model checking purposes.

At a more pragmatic level, a second important contribution is bringing the model transformation approaches based on graph grammars and informal approaches extending MOF, such as QVT, significantly closer; and providing tool support for defining model transformations, executing them, and model checking their properties. To the best of our knowledge, MOMENT2 is the first formal approach and tool supporting QVT-like model transformations and their formal analysis through model checking techniques.

In Section 2 a model of a distributed mutual exclusion algorithm is presented to illustrate the definition and verification of model transformations; in Section 3 
we introduce rewriting logic prerequisites and the algebraic semantics of MOF metamodels in MOMENT2; in Section 4 the semantics of the MOMENT2 model transformation language is given; in Section 5 model checking facilities are illustrated; in Section 6 we discuss related work, conclusions and future work.

\section{Modeling a Distributed MUTEX Algorithm}

A distributed mutual exclusion algorithm is used in operating systems and databases to ensure that a resource is never used by more than one process at a time. However, each request of a process for a resource must eventually be granted without running into a deadlock. In this section, we provide a metamodel-based version of a mutual exclusion algorithm [12].

In our approach, a model that conforms to the metamodel in Fig. 1. (a) represents the state of the system. Imagine this as the metamodel of a domainspecific language for visualizing and configuring processes and resources, where rules specify policies for how resources shall be assigned. In this metamodel, the Ring class contains some properties that are used for supporting fairness in the application of rules in Section 5. Fig. 1.(b) provides a model describing a deadlock state. Fig. 1. (c) provides an initial state model checked in Section 5.

We use the concrete syntax that was used in 12 for presenting the algorithm, where processes are drawn as black nodes and resources as light boxes. An edge from a process to a resource models a request. A solid edge in the opposite direction shows that the resource is currently held by the process. A dashed edge from a resource to a process asks the process to release the resource. Fig. 2 provides two sets of rules describing: (i) the mutual exclusion algorithm (ME) and (ii) the distributed deadlock detection mechanism (DDD).

Mutual exclusion ( $M E$ ). The system state is a cyclic list of processes linked by the next reference. For each resource there is a token, represented by an edge with a white flag, which is passed from process to process along the ring. If a process wants to use a resource, it waits for the corresponding token. Mutual

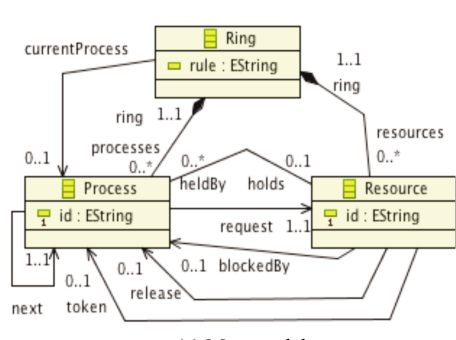

(a) Metamodel

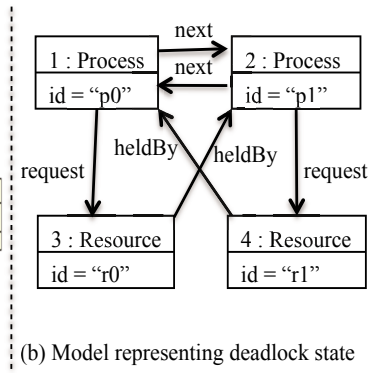

(b) Model representing deadlock state

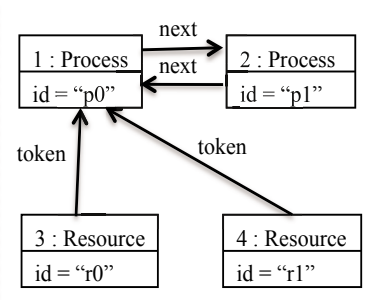

(c) Initial state

Fig. 1. (a) Metamodel $\mathscr{M}$. (b) Model representing a deadlock state. (c) Model representing an initial state for model checking purposes. 


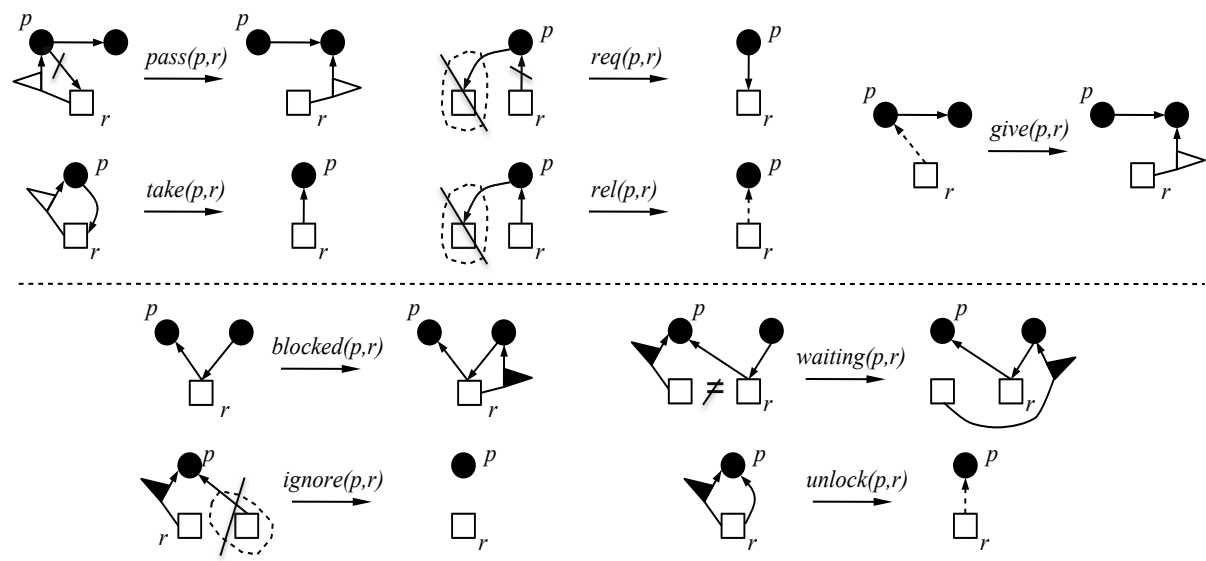

Fig. 2. Algorithms for mutual exclusion and deadlock detection

exclusion is ensured because there is only one token for each resource in the system. Among the ME rules, $\operatorname{pass}(p, r)$ describes that a process having the token may pass it to the next process in the ring, provided that it does not have a request on the corresponding resource. This negative application condition is visualized by the crossed-out request edge from the process to the resource. If a process wants to use a resource, it may generate a request. This is modeled by the rule $r e q(p, r)$, which is only applicable if the process does not have any requests yet, and if the particular resource is not used already by this process. If a process receives a token and there is a request for the resource, the process will choose the rule take $(p, r)$ replacing the token and the request by a heldBy edge from the resource to the process. When it has finished its task, the process may release its resource and give the token to the next process using $\operatorname{rel}(p, r)$ and give $(p, r)$. This will happen only when there are no pending requests for $\mathrm{r}$, which is modeled by a negative application condition at $\operatorname{rel}(p, r)$.

Distributed deadlock detection. In a model representing a state, a deadlock is represented as a cycle of request and heldBy edges. The distributed deadlock detection uses blocked messages, represented by edges with a black flag from a resource to a process, in order to detect cyclic dependencies. The blocked $(p, r)$ rule detects when a process requests a resource already held by another process and the rule unlock $(p, r)$ ensures that the resource will be released at some point.

We use the union of these two algorithms to show how relational in-place model transformations can be defined and model checked in MOMENT2. On the one hand, we model check the safety property MUTEX-safe: a resource cannot be held by two different processes, by means of reachability analysis of an invariant defined with a model pattern. On the other hand, we verify the liveness property MUTEX-live: each request of a resource by a process will eventually be granted, by means of model checking of LTL properties. 


\section{Preliminaries: Rewriting Logic and MOMENT2}

The key point of rewriting logic is to provide a general and flexible logical framework for concurrent systems, which are specified as rewrite theories so that their concurrent computation exactly corresponds to deduction by rewriting [1]. Specifically we specify a concurrent system as a rewrite theory $\mathcal{R}=(\Sigma, E, R)$ where: (i) $(\Sigma, E)$ is an equational theory, in some variant of equational logic, that specifies the system states as elements of the initial algebra $T_{(\Sigma, E)}$ associated to $(\Sigma, E)$ (see, e.g., [13), and where (ii) $R$ is a set of rewrite rules that describe in a parametric way all the one-step concurrent transitions in the system.

The underlying equational logic can be unsorted, many-sorted, order-sorted, or the more general membership equational logic (MEL) [13, which is the variant we adopt in this paper. It has the advantage of supporting expressive sorts, subsorts, and partiality. Its atomic sentences include not only equations $t=t^{\prime}$, but also memberships $t: s$, stating that term $t$ has sort $s$. For our purposes in this paper MEL has the additional advantage, as we further explain in Section 3.1. of providing the algebraic semantics for MOF metamodels on which the MOMENT2 tool is based. That is, given a MOF metamodel $\mathscr{M}$, its algebraic semantics is a MEL theory $\mathbb{A}(\mathscr{M})$. The models $M$ conformant with $\mathscr{M}$ then appear as elements of the initial algebra $T_{\mathbb{A}(\mathscr{M})}$. This gives us what we might call the static semantics of models in $\mathscr{M}$. The point of considering model transformations is that they can specify a dynamic semantics for models, in which each model $M$ is now viewed as a state (for example, a dynamic software architecture configuration), and model transformations become state transitions. It is precisely in this passage from the static to the dynamic semantics of models that rewriting logic is particularly helpful: as we explain in Section 4 a model transformation specifying dynamic model changes for models of a metamodel $\mathscr{M}$ can be precisely characterized as a rewrite theory extending the MEL theory $\mathbb{A}(\mathscr{M})$ that specifies the static semantics.

All this is of more than theoretical interest, because there are several highperformance implementations of rewriting logic. In particular, the Maude implementation [14] supports not only execution, but also verification of invariants and model checking of LTL properties, and has various other formal tools for verification purposes. This is heavily exploited in the MOMENT2 tool, where, as we further explain in Section 5. Maude is used as the underlying engine to model check invariants and LTL properties of model transformations.

We assume rewrite theories of the form $\mathcal{R}=(\Sigma, E \cup A, R)$, with $E, A$, and $R$ finite and where $A$ is a set of axioms, so that both the equations $E$ and the rules $R$ are applied modulo the axioms $A$. That is, we rewrite not just terms $t$ but rather $A$-equivalence classes $[t]_{A}$. The axioms $A$ are for example very important in the algebraic semantics $\mathbb{A}(\mathscr{M})$ of a metamodel $\mathscr{M}$, because the axioms $A$ of associativity, commutativity, and identity of set union exactly capture the graph-theoretic nature of a model $M$ in $\mathscr{M}$ as a set of objects linked by mutual references. Then, rewriting $M$ modulo such axioms exactly corresponds to graph rewriting, a correspondence discussed in [15] and systematically exploited in MOMENT2 as we explain in this paper. 
Furthermore, we assume the following executability properties of $\mathcal{R}=(\Sigma, E \cup$ $A, R)$ : (i) the equations $E$ are confluent and terminating modulo $A$, and (ii) the rules $R$ are coherent with $E$ modulo $A$. We refer to 14 for a detailed description of these properties and give here only an intuitive description of them. Confluence and termination of $E$ mean that the rules $E$ can be applied from left to right to always obtain a unique (modulo $A$ ) canonical form for any term. Coherence of $R$ with $E$ intuitively means that the strategy of first simplifying a term with the equations $E$ to canonical form and then rewriting it with $R$ is complete: no reachable states are missed by imposing this strategy. For tool support in Maude to verify confluence, termination, and coherence see [14].

\subsection{MOMENT2: MOF and Models}

MOMENT2 is based on a reflective, algebraic, executable specification of the

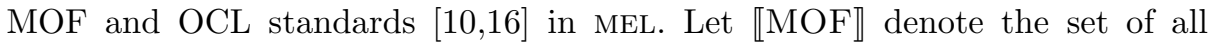
MOF metamodels $\mathscr{M}$, and let SpecMEL denote the set of all MEL specifications. The algebraic semantics of a MOF metamodel $\mathscr{M}$ is defined as a function $\mathbb{A}$ : $\llbracket \mathrm{MOF} \rrbracket \longrightarrow S p e c M E L: \mathscr{M} \mapsto \mathbb{A}(\mathscr{M}) . \mathbb{A}(\mathscr{M})$ provides a sort $M o d e l$, whose carrier $T_{\mathbb{A}(\mathscr{M}), \text { Model }}$ is defined by a membership axiom

\section{$M:$ Model if wellFormed $(M)=$ true}

that ensures that a model $M$ is semantically well-formed: identifiers are unique, objects are instances of object types of $\mathcal{M}$, there are no dangling edges, and the containment hierarchy, defined by means of composition associations, is preserved. $T_{\mathbb{A}(\mathscr{M}) \text {, Model }}$ defines the set of terms that represents models $M$ conforming to the metamodel $\mathscr{M}$, denoted $M: \mathscr{M}$. The MOF metamodels $\mathscr{M}$ that we consider involve the following relational constraints: inheritance relations, and composition and association relations with multiplicities (cardinalities, order and uniqueness). When a model $M$ conforms to a metamodel $\mathscr{M}, M$ implicitly satisfies these constraints by means of the aforementioned membership. For the metamodel $\mathscr{M}$ in Fig. 1.(a), the model depicted in Fig. 1.(b) can be defined as a term of sort Model in the $\mathbb{A}(\mathscr{M})$ theory as follows:

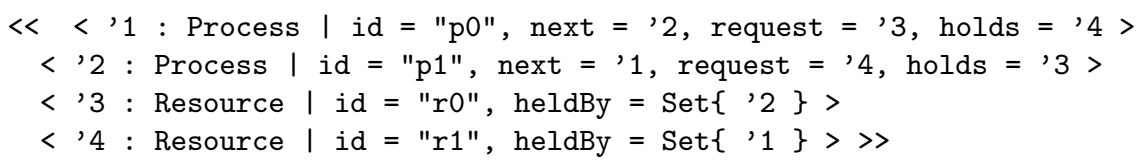

where the set of four tuples is formed by an associative and commutative union operator with empty syntax (juxtaposition), and each tuple < Oid : ClassName I Properties > represents an object that is typed with a specific object type of the corresponding metamodel. Objects are defined with properties of two kinds: attributes, typed with simple data types, and references, typed with object identifier types. Each property is defined by a pair (name = value). All the constructors that are used in the previous term are defined in the signature of the $\mathbb{A}(\mathscr{M})$ theory. The representation of models as algebraic terms is automatically generated by MOMENT2 from models in the Eclipse Modeling Framework (EMF) [17. A detailed definition of the mapping $\mathbb{A}$ can be found in 918 . 


\section{Rewriting Logic Semantics of Model Transformations}

MOMENT2's model transformation language supports the standards MOF, OCL and QVT: (i) MOF for specifying object types, (ii) OCL for manipulating attribute values, and (iii) QVT for specifying model patterns. In this paper we focus on endogenous, relational transformations, where the source and target metamodels are the same. A pair $(\mathscr{M}, \mathscr{T})$, of a MOF metamodel $\mathscr{M}$ and a MOMENT2 model transformation $\mathscr{T}$, represents a model transformation, whose semantics is formally defined by a rewrite theory $\mathbb{R}(\mathscr{M}, \mathscr{T})$ given by a semantic function $\mathbb{R}:$ SpecTransf $\rightarrow \operatorname{Spec} R L:(\mathscr{M}, \mathscr{T}) \mapsto \mathbb{R}(\mathscr{M}, \mathscr{T})$ such that $\mathbb{A}(\mathscr{M}) \subseteq \mathbb{R}(\mathscr{M}, \mathscr{T})$. The nondeterministic outcomes of a transformation $(\mathscr{M}, \mathscr{T})$ applied to an input model $M: \mathscr{M}$ are obtained by rewriting $M$ with the rules in $\mathbb{R}(\mathscr{M}, \mathscr{T})$.

In this section, we describe: (i) the concrete syntax of our model tranformation language, (ii) the semantics of an admissible model transformation $(\mathscr{M}, \mathscr{T})$ as a rewrite theory $\mathbb{R}(\mathscr{M}, \mathscr{T})$, and (iii) a notion of consistent model transformations that will be helpful for verification purposes. The MOMENT2 QVT syntax to specify $\mathscr{T}$ and the rewriting logic semantics $\mathbb{R}(\mathscr{M}, \mathscr{T})$ are illustrated by using the graph production rule $\operatorname{rel}(p, r)$ in Figure 3 as a running example.

\subsection{QVT-Based Syntax for Model Transformations}

Transformation. An in-place, relational transformation is specified as a pair $(\mathscr{M}, \mathscr{T})$, which is declared by providing a label, a domain and a set of model rewrites. The QVT notion of domain corresponds to a model that is used in the transformation. The transformation declaration of the example is defined as transformation mutexAlgorithm (model : mutex) $\{\ldots\}$, where mutexAlgorithm corresponds to the name of the model transformation, model corresponds to the domain variable, and mutex corresponds to an identifier for the metamodel.

Model Equation/Rewrite. Model transformation rules can be defined either as equations or as rewrites by the respective keywords eq or rl, respectively. The corresponding semantics is that they are respectively interpreted as equations in MEL or as rewrites in rewriting logic as explained in Section 3, A model transformation rule always consists of three elements: a label, a left-hand side (LHS) pattern and a right-hand side (RHS) pattern, where the LHS and RHS patterns correspond to collections of object template patterns in the QVT terminology, or to graph patterns in the graph tranformation terminology. Optionally, we can add a set of (possibly conditional) negative application conditions (NACs) to each model transformation rule and a global condition with the when clause. A complete definition of the model transformation language is provided in [19].

QVT Model Patterns. In a model equation/rewrite, model patterns are represented by using the QVT syntax for object patterns (object templates in the QVT specification). A model pattern is a collection of object patterns that are applied over a specific domain model. In our running example, we only have one domain 

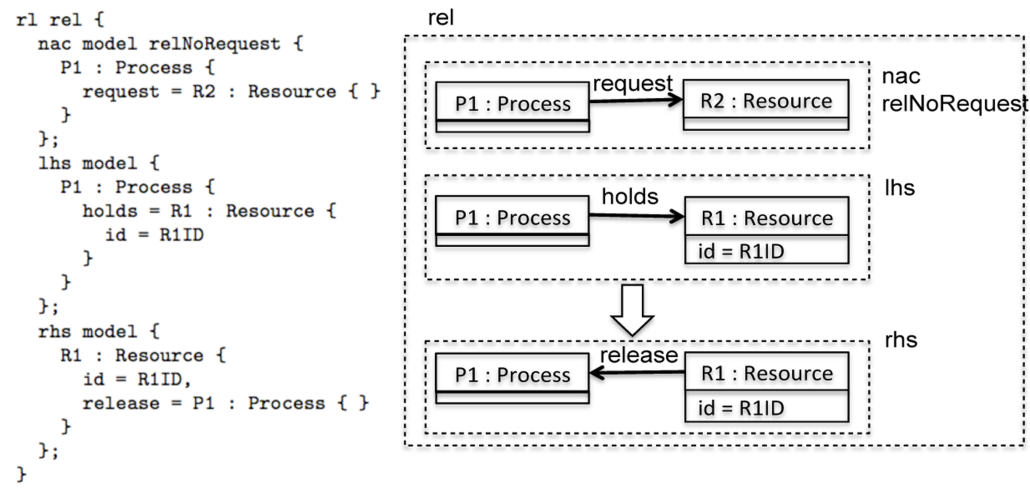

Fig. 3. Model rewrite $\operatorname{rel}(p, r)$ : textual format in MOMENT2 and graphical representation

called model. The LHS model pattern of the $\operatorname{rel}(p, r)$ model rewrite in Fig. 3 can be applied over a model that conforms to the metamodel in Fig. 1. (a). This is due to the transformation declaration above. In this model pattern, an object of type Process points to an object of type Resource through a holds reference. We also match the value of attribute id of the Resource object with the variable R1ID to illustate how attributes can be included in model patterns. This model pattern is shown as a graph pattern in Fig. 3 .

Conditions. In MOMENT2 a user can define rules with conditional and negative application conditions, which can be defined as boolean OCL expressions. In addition, OCL expressions can be used to query data in the NAC of a model equation/rewrite and to manipulate data in its RHS. That is, OCL expressions can be used to manipulate the shared variables that are matched either in a LHS pattern or in a NAC of a model transformation rule. See 9] for a detailed algebraic specification of OCL and [16] for some of its applications.

\subsection{Rewriting Logic Semantics of Model Transformations}

Let SpecTransf denote the set of pairs $(\mathscr{M}, \mathscr{T})$ where $\mathscr{M}$ is a metamodel and $\mathscr{T}$ is a MOMENT2 model transformation definition. The rewriting logic semantics is given by the function $\mathbb{R}:$ SpecTransf $\longrightarrow \operatorname{Spec} R L:(\mathscr{M}, \mathscr{T}) \mapsto \mathbb{R}(\mathscr{M}, \mathscr{T})$, where the rewrite theory $\mathbb{R}(\mathscr{M}, \mathscr{T})$ is such that $\mathbb{A}(\mathscr{M}) \subseteq \mathbb{R}(\mathscr{M}, \mathscr{T})$. However, not all model transformation specifications $(\mathscr{M}, \mathscr{T})$ correspond to valid model tranformations, because, as explained in Section 3, the associated rewrite theory $\mathbb{R}(\mathscr{M}, \mathscr{T})$ should satisfy reasonable executability requirements such as confluence and termination of equations, and coherence between equations and rewrites. We call a model tranformation $(\mathscr{M}, \mathscr{T})$ admissible iff $\mathbb{R}(\mathscr{M}, \mathscr{T})$ satisfies such executability requirements. In what follows we illustrate how the model rewrite $\operatorname{rel}(p, r)$ in Fig. 3, already specified in Section 4.1, is translated by $\mathbb{R}$ into a conditonal rewrite rule in the corresponding theory $\mathbb{R}(\mathscr{M}, \mathscr{T})$. 
Model equations/rewrites. In our example rewrite theory $\mathbb{R}(\mathscr{M}, \mathscr{T})$, there is an operator op mutexAlgorithm : Model $\rightarrow$ Model, corresponding to the transformation's name. The $\mathbb{R}$ function maps each model equation/rewrite in an admissible model transformation $(\mathscr{M}, \mathscr{T})$ to equations/rewrites in the theory $\mathbb{R}(\mathscr{M}, \mathscr{T})$. In the running example, model equations are mapped into equations that define the above operator mutexAlgorithm. Model rewrites are mapped to rewrites where this operator is the top symbol of the term that is rewritten. Within the mapping of a model equation/rewrite, the following steps are considered: $(i)$ the LHS model pattern becomes a term with variables $t\left(X_{1} \ldots X_{n}\right)$ that is used to match a model $M$, such that $M: \mathscr{M},($ ii $)$ the RHS of a model transformation rule becomes a sequence of operators that uses the variables $X_{1} \ldots X_{n}$ to perform atomic changes over a model $M$, and (iii) the when and such that clauses become boolean expressions used as conditions in the resulting equation/rewrite.

LHS Model Pattern. A collection of object templates that constitutes the LHS of a model equation/rewrite in a model transformation $\mathscr{T}$ is mapped to a term with variables. For example, the model pattern

lhs $\operatorname{model}\{\mathrm{P} 1: \operatorname{Process}\{$ holds $=\mathrm{R} 1:$ Resource $\{\mathrm{id}=\mathrm{R} 1 \mathrm{ID}\}\}\}$;

is mapped to the term with variables

< P10ID:0id : Process | holds = R10ID:0id, P1PS:PropertySet >

< R10ID:Oid : Resource | id = R1ID:String, R1PS:PropertySet > .

RHS Model Pattern. The RHS model pattern of a given model equation/rewrite is mapped to a sequence of equationally defined operators that perform atomic changes in a model. These changes correspond to the usual modeling primitives: to create a new object, to destroy an existing object, to set/unset attributes, or to update/remove references. These operators manipulate models in a consistent way so that dangling edges and orphan objects are automatically removed [19].

For the QVT expression of the RHS model pattern in the model rewrite $\operatorname{rel}(p, q)$ in Fig. 3 the corresponding RHS term is [[M] remove(P10ID:0id, "holds", R10ID:Oid)] update(R10ID:Oid, "release", P10ID:0id), where $M$ corresponds to the model that is matched in the LHS model pattern, the operator [M] op represents the application of an atomic change op over the model $\mathrm{M}$, and the variables that correspond to object identifiers are matched in the LHS model pattern, as shown above. In this example, the holds reference is removed from the object identified by P10ID:0id and the release reference is updated in the object with identifier R10ID:0id. A complete definition of the mapping is presented in [19] considering all possible combinations of atomic changes.

NACs and conditions. NACs in model transformation rules are compiled into equationally defined boolean functions as detailed in 19. These functions are used in the conditions of the equations and rules that are generated from model transformation rules checking whether a model pattern matching fails or not. Such that expressions are compiled into conditions for the equations that define 
the corresponding NAC function. When expressions are compiled into conditions for the equations or rules that are generated for model transformation rules. When and SuchThat clauses are constructed using OCL expressions that are compiled into terms as explained in 9 .

\subsection{Consistent Model Transformations}

The dynamic semantics of model-based systems can be given by means of model transformations $(\mathscr{M}, \mathscr{T})$, where the set of system states is a subset of the model type $\llbracket \mathscr{M} \rrbracket$ and transitions are defined by model rewrites in $\mathscr{T}$. The semantic mapping $\mathbb{R}$, when applied to admissible model transformations, plays a crucial role to model check invariants and LTL properties. It ensures that a model transformation $\beta$, defined by $\mathbb{R}(\mathscr{M}, \mathscr{T})$, always preserves the constraints of a metamodel $\mathscr{M}$ (types and reference multiplicities - cardinalities, order, uniqueness), when it is applied to a well-formed model $M: \mathscr{M}$, i.e., $\beta(M): \mathscr{M}$.

Theorem 1. An admissible model transformation $(\mathscr{M}, \mathscr{T})$ represents a model transformation $\beta$ in $\mathbb{R}(\mathscr{M}, \mathscr{T})$ that preserves the metamodel conformance relation, i.e., that is consistent w.r.t. the constraints in $\mathscr{M}$.

Proof (Sketch). Since $(\mathscr{M}, \mathscr{T})$ is assumed admissible, model equations in $\mathscr{T}$ are ground confluent 1 and terminating modulo structural axioms (associativity, commutativity and identity), model equations and rewrites are coherent and there are no free variables in the LHS, NAC and when expressions.

$\mathbb{R}$ maps model equations/rewrites in $(\mathscr{M}, \mathscr{T})$ to ordinary equations/rewrites in $\mathbb{R}(\mathscr{M}, \mathscr{T})$, where the RHS is given as a sequence of atomic changes performed by means of equationally defined operators, namely, new, destroy, set, unset, update and remove. In [19, we prove that the equations that define these operators are confluent and terminating. Such operators are completely defined by equations for admissible model transformations $(\mathscr{M}, \mathscr{T})$, so that these operators will never appear in the normal form that represents a model $M: \mathscr{M}$. Furthermore, the atomic changes that are performed by means of these operators for admissible model transformations $(\mathscr{M}, \mathscr{T})$ only produce well-formed models $M^{\prime}: \mathscr{M}$. This key consistency property is proved by structural induction in [19].

\section{Dynamic Analysis in MOMENT2}

Model Checking Model-Based Invariants. In a model-based system defined by an endogenous, relational model transformation $(\mathscr{M}, \mathscr{T})$, we are interested in checking invariants, that is, predicates that hold of a given initial state $M: \mathscr{M}$ and of all models $M^{\prime}: \mathscr{M}$ reachable from $M$ by means of state transitions produced by model rewrites. We can use Maude's breadth-first search for

\footnotetext{
${ }^{1}$ Rewrite steps with model equations make specific choices of names for the objects in the model and the choice might be different depending on the rewrite steps chosen. Graph-theoretically, different models may thereby represent the same abstract model up to renaming. We consider confluence of equations up to name isomorphism 19.
} 
this purpose by searching for a reachable state violating the invariant. Even if the number of reachable states is infinite, due to the breadth-first nature of the search, this gives a semidecision procedure for invariant violations. But if the reachable states are finite it becomes a decision procedure. We can define the negation of an invariant predicate by means of a pair $(P, C)$, where $P$ is a model pattern describing potentially "bad" states, and $C$ is a boolean predicate imposing additional semantic restrictions on the model pattern $P$. The compilation process then transforms the pair $(P, C)$ into a corresponding pair $\left(t, C^{\prime}\right)$, where $t$ is the pattern term with variables corresponding to $P$, and $C^{\prime}$ is a boolean condition involving the variables of $t$ that expresses condition $C$ at the term level. The original invariant $I_{\neg\left(t, C^{\prime}\right)}$ that $\left(t, C^{\prime}\right)$ negates is then the set-theoretic complement of the set of states that are instances of $t$ and satisfy $C^{\prime}$.

Given an initial state $M$ of sort Model in the theory $\mathbb{R}(\mathscr{M}, \mathscr{T})$, we write $\mathbb{R}(\mathscr{M}, \mathscr{T}), M \models I_{\neg\left(t, C^{\prime}\right)}$ to denote that all states reachable from $M$ satisfy the invariant $I_{\neg\left(t, C^{\prime}\right)}$. This is the case if and only if no state reachable from $M$ is an instance of $t$ that satisfies $C^{\prime}$, which can be semidecided by breadth-first search for an infinite number of reachable states, and can be decided by failure of such search if the set of reachable states is finite.

MOMENT2 uses Maude's search command for model checking invariant violations of the form $(P, C)$, with QVT model patterns $P$ and boolean OCL expressions $C$. In our mutual exclusion algorithm, we can verify that a resource will never be held by two different processes with the search command:

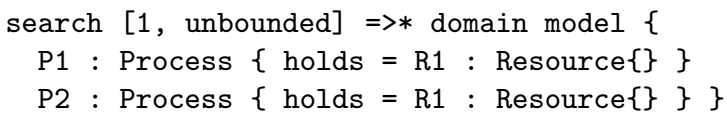

where the pair [1, unbounded] indicates that one single solution is searched and that all possible states are traversed, and $=>*$ states that we are applying zero, one, or more model rewrites. In [19], we explain the complete syntax of the command and its compilation to Maude's search command.

Model Checking LTL Properties. Given an admissible model transformation specified by $(\mathscr{M}, \mathscr{T})$ with semantics $\mathbb{R}(\mathscr{M}, \mathscr{T})$, we can verify Linear Temporal Logic properties over such a model transformation by using Maude's LTL model checker. In MOMENT2, we enable the use of model predicates in LTL formulas by defining a set $\mathcal{D}$ of model predicates for $(\mathscr{M}, \mathscr{T})$ by means of: $(i)$ possibly parametric model predicate symbols $\mathscr{P}$, and $(i i)$ model-based equations $E_{\mathscr{P}}$ defining the satisfaction relation of a predicate in a model. As an example, the satisfaction of a parametric model predicate requests $(P, R)$, where $P$ and $R$ are string parameters, can be defined by means of the equation

domain model $\{\mathrm{P} 1: \operatorname{Process}\{\mathrm{id}=\mathrm{P}$, request $=\mathrm{R} 1: \operatorname{Resource}\{\mathrm{id}=\mathrm{R}\}\}$

$\mathrm{I}=\operatorname{requests}(\mathrm{P}, \mathrm{R})=\operatorname{true}$

stating that the predicate requests $(\mathrm{P}, \mathrm{R})$ is satisfied in a state $M$, when there is a process with id $\mathrm{P}$ that requests a resource with id $\mathrm{R}$ in $M$. Predicate parameters can be either constants or variables that are matched with the model pattern. 
Let SpecPred denote the set of specifications of sets of predicates $\mathcal{D}$ that can be defined for an admissible model transformation $(\mathscr{M}, \mathscr{T})$. We define a function $\mathbb{A}:$ SpecPred $\longrightarrow$ SpecMEL that maps a set $\mathcal{D}$ of model predicates to a MEL theory defining the state predicates, which are used as propositions in LTL formulae as explained in [14]. For the aforementioned equation, the following equation is generated:

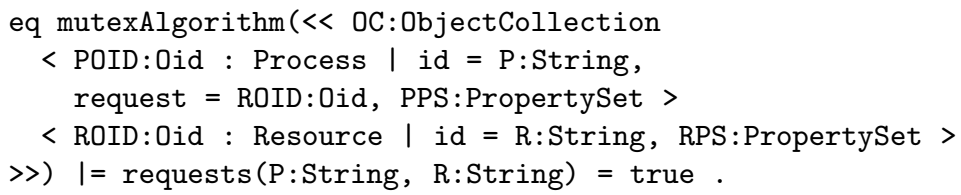

The following equation defines the satisfaction of the state predicate heldBy $(R$, $\mathrm{P}$ ), specifying when a resource with id $\mathrm{R}$ is held by a process with id $\mathrm{P}$ :

domain model $\{\mathrm{P} 1: \operatorname{Process}\{i d=\mathrm{P}, \operatorname{holds}=\mathrm{R} 1: \operatorname{Resource}\{\mathrm{id}=\mathrm{R}\}\}\}$ $I=\operatorname{heldBy}(R, P)=\operatorname{true}$

A model transition system defined by a meaningful model transformation $(\mathscr{M}, \mathscr{T})$ and a set $\mathcal{D}$ of model predicates for $(\mathscr{M}, \mathscr{T})$ is formally defined as a rewrite theory as follows: $(i)(\mathscr{M}, \mathscr{T})$ is mapped to the rewrite theory $\mathbb{R}(\mathscr{M}, \mathscr{T})$, and $(i i) \mathcal{D}$ for $(\mathscr{M}, \mathscr{T})$ is mapped to the equational theory $\mathbb{A}(\mathcal{D})$ that extends $\mathbb{R}(\mathscr{M}, \mathscr{T})$ with model predicate equations. The rewrite theory $\mathbb{R}(\mathscr{M}, \mathscr{T}) \cup \mathbb{A}(\mathcal{D})$ defines a Kripke structure $\mathcal{K}(\mathbb{R}(\mathscr{M}, \mathscr{T}) \cup \mathbb{A}(\mathcal{D}))$ as explained in [14], where the transition relation corresponds to one-step model rewrites in $\mathbb{R}(\mathscr{M}, \mathscr{T})$.

In this way, we enable the use of model predicates as propositions in LTL formulas in Maude's model checker. In the example, we want to verify that each process that requests a resource is always eventually served. This can be specified with the LTL formula [] (requests ("po", "ro") -> <> heldBy ("ro", "p0")). This property can be model checked in Maude by means of the command

red modelCheck ( mutexAlgorithm(model),

[] (requests ("p0", "r0") $\rightarrow<$ heldBy("r0", "p0")) ) .

By using the model in Fig. 1.(c) as initial state, Maude's model checker found a path in which the property is violated. In particular, the two processes $P 0$ and $P 1$ request the resource $R 0$ and the pass rule is henceforth applied over the resource $R 1$. This is due to the fact that the rules are not applied in a fair way, i.e., all rules are not equally applied in all possible paths. This problem can be solved by forcing the application of the take rule. In the metamodel in Fig. 1.(a), we have added the properties rule and currentProcess to the Ring class. The rule property indicates which rule has been applied and the currentProcess property indicates which process has been activated by the application of a rule. In the model transformation, we can modify the model rules so that each rule updates the rule and currentProcess in the Ring object as in the take rule:

rl take $\{$ lhs model $\{\mathrm{RG}: \operatorname{Ring}\{\} .$.$\} ;$

rhs $\operatorname{model}\{R G: \operatorname{Ring}\{r u l e=$ "take", $\operatorname{currentProcess}=$ P1 : Process \{\}$.\} ;$. 
where we only show the elements that are needed to ensure fairness. Two more model predicates are needed to check when the take rewrite can be applied (enabled-take) and when it has already been applied (take):

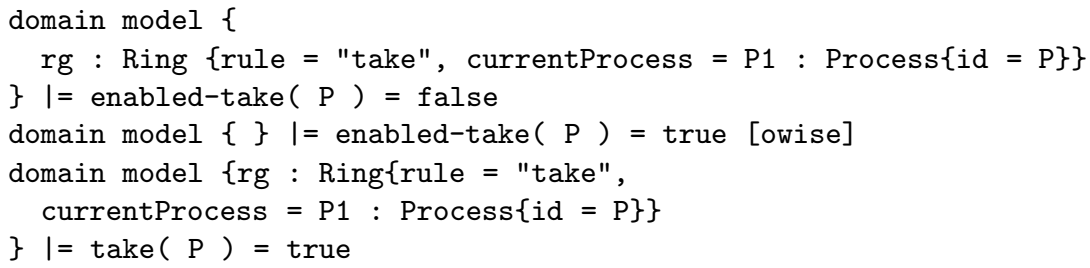

The MUTEX-live property can be model checked for the modified system by encoding the fairness of the take rule in the LTL formula as follows:

([] <> enabled-take ( "p0" ) $\rightarrow$ [] $<$ take ( "p0" )) $\rightarrow$

[] (requests("p0", "ro") $\rightarrow<$ heldBy("ro", "p0"))

\section{Related Work, Conclusions and Future Work}

This work has brought closer the algebraic and graph-based approaches to models and model transformations. In this sense, it continues a line of work on algebraic approaches to graphs and graph rewriting including [1 20 21 22 15]. The closest papers to the approach presented here are [22, and particularly [15], which makes explicit the relationship of graph rewriting with rewriting logic. However, our approach has some novel features not treated in previous work, which express the kinds of graph used in model-based software engineering. Specifically, we support different kinds of references, namely, ordinary references, and containment references, which have a different semantic treatment.

Several approaches based on model-checking provide automated procedures for formal verification of model-based systems. On the one hand, model-checking tools with specific support for graph transformations are particularly interesting due to the graph-theoretic nature of models. GROOVE 23] is a graph-based analysis tool that provides model checking for LTS whose states are graphs. Augur [24] is an analysis tool based on the translation of graph transformations to Petri nets and the application of Petri net analysis techniques. On the other hand, generic model checkers have been used, such as SPIN, in approaches such as CheckVML [25]. In [26], Maude is used as a programming language for encoding Atom3 visual graph transformations [27] as Maude system modules so that the state space of a graph transformation system can be examined.

On the other hand, there are fewer approaches that enable the verification of QVT-like model transformations, as opposed to graph transformations. A notion of graph with containments is used in 7. to encode model transformations as graph transformations, and to analyse termination and confluence of model transformations in the algebraic graph transformation environment AGG 28 . Another approach 29], based on Alloy, provides the analysis of the consistency of model transformations w.r.t. the metamodel conformance relation, which is 
ensured by construction in MOMENT2. Our approach focuses on the formalization of QVT-like model transformations in rewriting logic by reusing the theory of graph transformation systems. In this way, MOMENT2 enhances the application of Maude's techniques for reachability analysis and LTL model checking to model transformations by considering MOF modeling primitives, such as containment relationships, not directly supported in graph-based approaches. At the same time, our approach provides the rewriting logic semantics of graph transformations so that the computational semantics of graph rewriting is given by term rewriting, by considering production rules either as equations or as rewrites. This semantics is directly supported by the MOMENT2 tool [30, an Eclipse plugin in which model transformations between EMF metamodels can be conveniently defined, executed, and efficiently model checked.

Much work remains ahead, including: a closer study of the expressiveness of the MOMENT2 model transformation language and its applications for defining the formal semantics of popular transformation languages such as ATL [4] or QVT [5], and for defining graph-theoretic notions, such as graph constraints and related logics [31] and triple-graph grammars [32]; a further study of the rule/equation distinction for model transformation rules and symmetry reduction techniques for state reduction purposes in model checking; and the formal analysis of MOF domain-specific languages such as real-time DSLs.

Acknowledgments. We cordially thank Francisco Durán for his kind help with the CRC and MTT Maude tools. We also thank the anonymous reviewers for their helpful comments and suggestions. This work has been partially supported by the NSF Grant IIS-07-20482, by the project META TIN2006-15175-C05-01, and by the project SENSORIA, IST-2005-016004.

\section{References}

1. Ehrig, H., Ehrig, K., Prange, U., Taentzer, G.: Fundamentals of Algebraic Graph Transformation. Springer, Heidelberg (2006)

2. Ehrig, H., Montanari, U., Kreowski, H.J., Rozenberg, G., Kreowski, H.J.: Handbook of Graph Grammars and Computing by Graph Transformations, vol. 3. World Scientific Publishing Company, Singapore (1999)

3. OMG: Meta Object Facility (MOF) 2.0 Core Specification (ptc/06-01-01) (2006)

4. ATLAS Group: ATL web site (2008), http://www.eclipse.org/m2m/atl/

5. OMG: MOF 2.0 QVT final adopted specification (ptc/07-07-07) (2007)

6. Ehrig, H., Engels, G., Kreowski, H.J.: Handbook of Graph Grammars and Computing by Graph Transformation, vol. 2. World Scientific Publishing Company, Singapore (1999)

7. Biermann, E., Ermel, C., Taentzer, G.: Precise Semantics of EMF Model Transformations by Graph Transformation. In: Czarnecki, K., Ober, I., Bruel, J.-M., Uhl, A., Völter, M. (eds.) MODELS 2008. LNCS, vol. 5301. Springer, Heidelberg (2008)

8. Mens, T., Gorp, P.V.: A taxonomy of model transformation. Electr. Notes Theor. Comput. Sci. 152, 125-142 (2006)

9. Boronat, A.: MOMENT: a formal framework for MOdel manageMENT. PhD in Computer Science, Universitat Politènica de València (UPV), Spain (2007), http://www.cs.le.ac.uk/ aboronat/papers/2007_thesis_ArturBoronat.pdf 
10. Boronat, A., Meseguer, J.: An Algebraic Semantics for MOF. In: Fiadeiro, J.L., Inverardi, P. (eds.) FASE 2008. LNCS, vol. 4961, pp. 377-391. Springer, Heidelberg (2008)

11. Meseguer, J.: Conditional rewriting logic as a unified model of concurrency. Theoretical Computer Science 96(1), 73-155 (1992)

12. Heckel, R.: Compositional verification of reactive systems specified by graph transformation. In: Astesiano, E. (ed.) FASE 1998. LNCS, vol. 1382, pp. 138-153. Springer, Heidelberg (1998)

13. Meseguer, J.: Membership algebra as a logical framework for equational specification. In: Parisi-Presicce, F. (ed.) WADT 1997. LNCS, vol. 1376, pp. 18-61. Springer, Heidelberg (1998)

14. Clavel, M., Durán, F., Eker, S., Lincoln, P., Martí-Oliet, N., Meseguer, J., Talcott, C.: All About Maude - A High-Performance Logical Framework. LNCS, vol. 4350. Springer, Heidelberg (2007)

15. Meseguer, J.: Rewriting logic as a semantic framework for concurrency: a progress report. In: Sassone, V., Montanari, U. (eds.) CONCUR 1996. LNCS, vol. 1119, pp. 331-372. Springer, Heidelberg (1996)

16. Boronat, A., Meseguer, J.: Algebraic Semantics of OCL-constrained Metamodel Specifications. Technical Report UIUCDCS-R-2008-2995, UIUC (2008), http:// www.cs . uiuc. edu/research/techreports . php? report=UIUCDCS-R-2008-2995

17. Eclipse Organization: The Eclipse Modeling Framework (2007), http://www.eclipse.org/emf/

18. Boronat, A., Meseguer, J.: An algebraic semantics for MOF. Technical Report CS-08-005, University of Leicester (2008), http://www.cs.le.ac.uk/people/ aboronat/papers/boMe-mof-apps.pdf

19. Boronat, A., Heckel, R., Meseguer, J.: Rewriting Logic Semantics and Verification of Model Transformations. Technical Report CS-08-004, University of Leicester (2008), http://www.cs.le.ac.uk/people/aboronat/papers/boHeMe-rl-mt.pdf

20. Bauderon, M., Courcelle, B.: Graph expressions and graph rewriting. Math. Systems Theory 20, 83-127 (1987)

21. Corradini, A., Montanari, U.: An algebra of graphs and graph rewriting. In: Curien, P.-L., Pitt, D.H., Pitts, A.M., Poigné, A., Rydeheard, D.E., Abramsky, S. (eds.) CTCS 1991. LNCS, vol. 530, pp. 236-260. Springer, Heidelberg (1991)

22. Raoult, J.C., Voisin, F.: Set-theoretic graph rewriting. In: Ehrig, H., Schneider, H.J. (eds.) Dagstuhl Seminar 1993. LNCS, vol. 776, pp. 312-325. Springer, Heidelberg (1994)

23. Rensink, A.: The GROOVE simulator: A tool for state space generation. In: Pfaltz, J.L., Nagl, M., Böhlen, B. (eds.) AGTIVE 2003. LNCS, vol. 3062, pp. 479-485. Springer, Heidelberg (2004)

24. König, B., Kozioura, V.: Augur 2-a new version of a tool for the analysis of graph transformation systems. ENTCS, vol. 211, pp. 201-210. Elsevier, Amsterdam (2008)

25. Schmidt, Á., Varró, D.: CheckVML: A Tool for Model Checking Visual Modeling Languages. In: Stevens, P., Whittle, J., Booch, G. (eds.) UML 2003. LNCS, vol. 2863, pp. 92-95. Springer, Heidelberg (2003)

26. Rivera, J.E., Guerra, E., de Lara, J., Vallecillo, A.: Analyzing rule-based behavioral semantics of visual modeling languages with maude. In: SLE (2008)

27. de Lara, J., Vangheluwe, H., Alfonseca, M.: Meta-modelling and graph grammars for multi-paradigm modelling in AToM $^{3}$. Software and System Modeling 3(3), 194209 (2004) 
28. AGG Homepage (2008), http://tfs.cs.tu-berlin.de/agg/

29. Anastasakis, K., Bordbar, B., Küster, J.M.: Analysis of Model Transformations via Alloy. In: Giese, H. (ed.) MODELS 2008. LNCS, vol. 5002. Springer, Heidelberg (2008)

30. MOMENT2 (2008), http://www.cs.le.ac.uk/people/aboronat/tools/moment2

31. Orejas, F., Ehrig, H., Prange, U.: A logic of graph constraints. In: Fiadeiro, J.L., Inverardi, P. (eds.) FASE 2008. LNCS, vol. 4961, pp. 179-198. Springer, Heidelberg (2008)

32. Schürr, A.: Specification of graph translators with triple graph grammars. In: Mayr, E.W., Schmidt, G., Tinhofer, G. (eds.) WG 1994. LNCS, vol. 903, pp. 151-163. Springer, Heidelberg (1995) 